\title{
A sustainable solar and carbon-based economy: mitigating climate risk, promoting energy justice and stimulating human prosperity
}

Keywords: solar energy, human prosperity, carbon management, sustainable fuels, carbon composites

Abbreviations: $\mathrm{CO}_{2}$, carbon dioxide; GHG, greenhouse gas; $\mathrm{H}_{2} \mathrm{O}$, water; $\mathrm{MT} / \mathrm{yr}$, million (metric) tons per year; GT/yr, billion (metric) tons per year; PPMV, parts per million by volume; BECCS, bio energy with carbon capture and sequestration; DAC, direct air capture

\section{Introduction}

Is it time to start thinking differently about carbon dioxide $\left(\mathrm{CO}_{2}\right)$ and about the role of photo-energy in the socio-technical industrial complex? The reason I say this is because we are running out of time and progress rests on creating value as long as there is a substantial cost or perceived cost to the economy or to specific sectors of the economy, there will be resistance. What we are doing now is degrading essential natural ecosystems and changing the composition of both the atmosphere and the ocean and deferring the costs from these actions to future generations and to vulnerable populations. We also know this is patently unsustainable and that we need to start reversing course, restoring the carbon balance, and regenerating degraded ecosystems. However, the headwinds remain strong. Nevertheless, in the electricity sector, progress is significant and seemingly unstoppable, transportation, manufacturing, and buildings are not transitioning fast enough.

In trying to deal with climate risk, $\mathrm{CO}_{2}$, renewable energy, and the economy, the conversation leans heavily towards decarbonizing and mostly on producing carbon free electricity. In other sectors, the conversation and innovations lean heavily on finding efficienciesreducing the carbon intensity, but not yet a major focus on getting to zero net lifecycle emissions. Furthermore, we do not often make a clean distinction between primary and secondary energy sources. In not doing so, society may be missing an opportunity to expand the innovation and solution space, to turn what is now a liability into an asset, and to have solar (or photon) energy penetrate every aspect of the energy system to get to net zero and even net negative. The latter (net negative) gives society an opportunity to both accelerate the rate of decreasing net emissions and to mitigate the substantial risk that we will overshoot the Paris Climate Agreement target of $<450$ parts per million by volume (ppmv) $\mathrm{CO}_{2}$ in the atmosphere. ${ }^{1}$ We expand some on these thoughts below.

Current practice of emitting very large quantities of $\mathrm{CO}_{2}^{2}$ and other greenhouse gases $(\mathrm{GHG})$ is a large-scale geo-engineering experiment with the only planet we live on. I am not saying anything new. However, one obvious action would be to begin directly removing $\mathrm{CO}_{2}$ from the atmosphere, ${ }^{3}$ much as the biosphere does, but do so more rapidly by artificial means. The atmosphere is very effective at dispersing $\mathrm{CO}_{2}$ emissions from any source. Therefore, it is feasible to deliver a continuous and uniform supply of $\mathrm{CO}_{2}$ from an airstream to
Volume I Issue I - 2017

\author{
Stechel EBc \\ ASU Light Works and School of Molecular Sciences, Arizona \\ State University, USA
}

Correspondence: EB Stechel, ASU Light Works and School of Molecular Sciences, Arizona State University, Tempe, AZ, USA, Tel +00+0 I-480-965-1657, Fax 00+ I-505-903-6706, Email ellen.stechel@asu.edu

Received: June 27, 2017 | Published: July 17, 2017

any direct air capture (DAC) site, thus having the potential to avoid arable land. In addition, placement of such capture sites could be in sun rich arid or semi-arid regions and limit concern for impact to food systems or biodiversity.

While not a "silver bullet," and not an alternative to decarbonizing primary energy resources, it is hard to understand why there is not a significant effort supported by governments to develop DAC, based on using carbon-free primary energy. The reasons for this inattention are seemingly because first it is expected to be too expensive $e^{4}$ and second it has been lumped together with solar radiation management and iron fertilization in the ocean-the latter category referred to as geo-engineering. ${ }^{5}$ However, I do find it ironic that we would consider pulling $\mathrm{CO}_{2}$ back out of the atmosphere as geo-engineering. To my way of thinking, dumping uncontrolled amounts of $\mathrm{CO}_{2}$ and other GHGs is the real geo-engineering, not removing these gases. Pulling some of the excess back out of the atmosphere is just undoing what was a bad idea to begin with. Hence, I contend it is natural and most certainly not geo-engineering to reverse course, as long as we are only removing from the excess and especially if all we are doing is slowing the growth. Below, we will return to the question of negative emissions, where we are not slowing growth, but actually having the goal to decrease the concentration of $\mathrm{CO}_{2}$ in the atmosphere. Nevertheless, if we are able to mine the atmosphere for the excess $\mathrm{CO}_{2}$ using energy from the sun to overcome the entropy, we will have only taken a step towards restoring the carbon balance. However, we still have to do something with that mined $\mathrm{CO}_{2}$ and ideally to create value. It is time to turn our innovation system in a serious way to finding creative and value-add uses for that $\mathrm{CO}_{2}{ }^{6,7}$ and embrace innovations to mine $\mathrm{CO}_{2}$ from the atmosphere (or the ocean).

\section{Primary energy}

Today, the dominant source of what we recognize as primary energy is fossil fuels. Fossil resources also contribute materially to chemicals and materials in society. Here, I will focus my comments on 
solar as the likely dominant primary energy source of the future, first, because this is a journal on photo-energy, and second because solar is here today and highly scalable. The open question is not will this transition occur (from fossil dominated to solar dominated, possibly nuclear dominated) but when. That said, it would be presumptuous to predict the future, although fossil resources are finite so there is no choice. However and unfortunately, they are not finite enough that resource scarcity will be the cause of the transition. Furthermore, the proven resources contribute a great deal to the valuation of the fossil fuel industry, leading to strong competing motivations not to leave it in the ground.

Despite it being customary to consider fossil energy as a primary energy source, because nature has prepared it for us and because we can just dig it up; it is a natural resource, but not actually primary energy. It is more appropriate and instructive to recognize that conventional fossil fuels are in fact, "stored (ancient) sunlight" in the form of energy dense, sequestered both carbon and hydrogen, which nature took millions of years to produce and modern civilization is consuming in only centuries. Dukes ${ }^{8}$ has estimated that it takes over three million units of sunlight to make one unit of petroleum. There is abundant and unlimited sunlight, but the amount of solar power that falls on the earth is nevertheless finite, limiting the production rate when converting sunlight to secondary energy either in the form of new energy carriers or process heat.

\section{Secondary or tertiary energy sources}

When it comes to secondary energy, then we cannot claim to know the future composition of the secondary or tertiary energy portfolio. Much of current conversation focuses on electricity as the almost exclusive energy carrier and tertiary energy then derived from electricity. Nevertheless, I argue here that there is value in seeking a diversity of energy carriers, including liquid hydrocarbons, hydrogen, carbon monoxide, ammonia, and alcohols as long as they are derived from carbon free primary energy resources, such as solar, i.e., photon, energy or another carbon-free source. Diversity generally leads to more resiliency, more robustness, less strain on resource limits, and greater overall success. Below, we will talk more about the advantages of liquid hydrocarbons, and why we might want to keep them as an integral part of the energy portfolio (just not from fossil energy).

\section{Recycling carbon dioxide}

$\mathrm{CO}_{2}$ and water are simply the energy-depleted, oxidized form of the carbon and hydrogen making up a hydrocarbon. Thus, we might consider reframing the problem as a techno-economic challenge to use solar energy (as nature did) and reverse combustion, recycle $\mathrm{CO}_{2}$, now fast enough to match consumption. Whether, we emulate nature by producing advanced biofuels or by producing synthetic fuels directly from sunlight, $\mathrm{CO}_{2}$, and water (without biomass as an intermediary), to be sustainable, at a minimum we must "process intensify" over nature's path to fossil. Advanced biofuels (land-based cultivation, harvesting, and industrial processing) use approximately 100 or more units of sunlight and significant amounts of fresh water resources per unit of fuel (where the unit is one of power, energy per unit time.) Microalgae biofuels (aquatic-based cultivation, harvesting, dewatering, and industrial processing) can avoid fresh water and might get to 50 units of sunlight and potentially less if there is an additional form of energy rich nutrients as in waste water treatment or waste to energy. Synthetic pathways use purely engineered systems and aim to use no more than about 10 units. Within each of these three major pathways, there exists a large diversity of opportunities and approaches. Furthermore, in addition to making fuels (gasoline, diesel, jet,) blends (ethanol/ gasoline up to $85 / 15 \%$ or higher alcohol blends) and energy carriers (e.g., hydrogen, carbon monoxide, methanol), each can be used as feedstock. Depending on the product produced and what happens to that product at end of life, the lifecycle can be low to neutral in net $\mathrm{CO}_{2}$ released and even net negative. Some interesting applications are low volume, but some products could grow to significant scale, if they could be produced cost competitively: for example, plastics carbonate ceramics, carbon fibers, and carbon composites. Furthermore, once the innovation space becomes better recognized and if research, development, and demonstration incentivized, the creativity engine that would be unleashed could lead to a range of other possibilities, some that we cannot imagine today.

For example, synthetic solar thermo chemical fuel processes can convert solar energy, excess $\mathrm{CO}_{2}$, and low quality water into gasoline, diesel, and aviation fuel-fuels that are compatible with the existing energy infrastructure. The solar thermo chemical approach, which is most appropriate for desert regions with copious direct normal solar insolation, is just one of several pathways to recycle $\mathrm{CO}_{2}$ back into fuel at rates considerably faster and more efficiently than the biosphere naturally captures and fixes $\mathrm{CO}_{2}$ from the atmosphere, and with fewer scale limitations. We expect that we will need all approaches, high and low temperature electrolysis, photo-electrochemical, advanced water splitting and then direct reaction of hydrogen and $\mathrm{CO}_{2}$, and considerable learning to get both scale and affordability of the most promising of the approaches. Fortunately, scale and affordability are synergistic. Generally, for every doubling of production, there are concurrent cost reductions.

Thus, an underexplored emerging strategy is to develop solar technologies that recycle-rather than bury-waste $\mathrm{CO}_{2}$ into new supplies of liquid hydrocarbon fuels and ultimately drawing on (mining) the excess $\mathrm{CO}_{2}$ in the atmosphere as the sustainable source of carbon. In so doing, the atmosphere provides a unique service of free storage and transport between source and processing facility. In addition to fuels, producing carbon-based structural materials, electrodes, and coatings could be an exciting opportunity, as it is becoming evident that negative emissions will likely be required. Furthermore, carbon is more versatile than virtually any element on the periodic table, contributing to more compounds than any other element (aside from hydrogen) with a large range of properties, new forms still being discovered, great enthusiasm, strong or soft, flexible, and non-corrosive. In other words, carbon can contribute in many applications due to its unique properties.

\section{What do we mean by scale?}

Not many material flows are measured on the scale of giga tons (GT $=10^{9}$ metric tons $)^{9}$ or GT per year (GT/yr.) Nevertheless, the atmosphere has $\sim 7.75$ million GT. At 400 ppmv, $\mathrm{CO}_{2}$ weighs in at $\sim 3100$ GT. Pre-industrial the $\mathrm{CO}_{2}$ in the atmosphere was $\sim 2200$ GT. Global $\mathrm{CO}_{2}$ emissions (from fossil fuel, cement, and land use changes) is nearly $40 \mathrm{GT} / \mathrm{yr}$. Land and vegetation uptakes $\sim 35 \%$ of these emissions and the oceans uptake $\sim 25 \%$. Hence, approximately $45 \%$ of the emissions remain in the atmosphere for a very long time. Proven fossil reserves have the potential to release additional cumulative emissions of $\sim 2800$ GT. However, in order to meet the goal from the 2015 Paris Climate Agreement, society can emit no more than a cumulative $\sim 500-900$ GT. To date cumulative emissions (between 
1750 and 2011) has been $204310 \mathrm{GT} \mathrm{CO}_{2}$ and the increase in the atmosphere has been $88035 \mathrm{GT} \mathrm{CO}_{2}$. To keep the remaining emissions below $900 \mathrm{GT}$, the global economy would need to reduce $\sim 4.25 \%$ year of year, which would give a decrease in net emissions of $30 \%$ in eight years and $50 \%$ in 16 years. Such decreases will be quite challenging in the face of population growth and economic development.

Given the scale of the problem, it is worth considering the application scale. Thus, for the purposes here, we consider something niche if the scale is unlikely to be more than 100 million metric tons per year (100 MMT/yr) and scalable if it will approach one billion metric tons per year (1000 MMT/yr or $1 \mathrm{GMT} / \mathrm{yr}$,) each by the end of the century. More than $100 \mathrm{MMT} / \mathrm{yr}$ and less than $1000 \mathrm{MMT} /$ yr is significant. Indeed, any scale can be important, if production of the product leads to learning and cost reductions that in turn enable products of a larger scale. One particular product that may fall in this niche to intermediate scale, are carbon-based electrodes for the growing product line of electrochemical devices; e.g., batteries, electrolyzers to split water and make hydrogen, electrochemical reduction of $\mathrm{CO}_{2}$, fuels cells, to name a few.

\section{A new carbon asset-based economy}

Since the 2015 COP21 Paris Climate Agreement, there is a new conversation about negative carbon. Renewable carbon-based fuels, even with high carbon atom efficiency from $\mathrm{CO}_{2}$ to fuel, are going to just close the cycle and at best be net neutral. However, in the spirit of seeking ways sustainably to turn $\mathrm{CO}_{2}$ into value, sequestering it in deep saline aquifers, incurs costs and provides no product value. Disposal has value to society by keeping it out of the atmosphere, the ocean and keeping it from wreaking havoc with the climate system. Hence, disposal in substantial quantities might regrettably be required. Nevertheless, greater value would accrue by turning the $\mathrm{CO}_{2}$ into tangible products.

Disappointingly, there has been lack of agreement and even controversy surrounding negative emissions. ${ }^{10,11}$ However, that discussion has narrowly focused on bioenergy plus carbon capture and sequestration (BECCS) and the risk of "banking" on an unproven technology at scale. Additionally, the controversy is mostly about how feasible (technologically, politically, economically, and socially) is it not to exceed the remaining carbon budget, or in other words not "overshooting" the target. Notwithstanding that controversy, it seems that we will need a comprehensive multi-prong strategy that would aggressively pursue energy efficiency; de-carbonization of primary energy; adaptation (as even at 1.5-2C it will be necessary to manage adverse impacts) $\mathrm{CO}_{2}$ capture, reuses, and recycles (transforming excess $\mathrm{CO}_{2}$ into valuable products) and lastly capture and disposal of what cannot be productively put into use. In addition, we will need to be prepared potentially to manage an "overshoot" scenario.

Hence, we envision a highly robust, resilient, scalable, and sustainable new carbon asset-based economy, with a range of tangible products that have carbon as a major component, where that carbon comes from $\mathrm{CO}_{2}$ that has been in the atmosphere or ocean in recent history as opposed to ancient carbon that has yet to be mobilized from fossil resources. This would include, but not be exclusive to, a bio-economy. In the near-term, some of that carbon may come from stationary sources and hence some old carbon, but ultimately will likely have to come indirectly from biomass and from emissions from bio-factories or directly by mining the atmosphere and/or the ocean. If modern sunlight is the primary energy source, then fuels produced in this new carbon asset-based economy can be net neutral, carbon-based material products (non-fuel and non-biodegradable)can lead to net negative, and some carbon can be used to restore degraded soils (as in biochar and adding to carbon stocks in the biosphere,) also leading to net negative.

Today, current thinking on a new carbon economy evokes thoughts of emissions trading and buying and selling carbon offsets. ${ }^{12}$ Tomorrow, we hope that it is a robust materials-based, tangible assetbased economy consisting of soil amendments, advanced biofuels, synthetic liquid hydrocarbons (solar fuels) plastics, carbonate ceramics, carbon composites, carbon electrodes, fertilizers, and other products made from $\mathrm{CO}_{2}$ mined from the atmosphere or the ocean.

\section{Do liquid hydrocarbon fuels have a future -are they still a good option?}

We ask the question as the reader might be asking: could liquid hydrocarbon-based fuel remain a viable and sustainable option in large quantities and if they could, should they? Often overlooked, liquid hydrocarbon fuels are unrivaled in the rate of delivery to on-board, usable energy storage. They are also unsurpassed in having high energy densities accommodating both space and weight requirements. Consequently, there are no credible alternatives for air, heavy-duty, or commercial ocean applications or end users must accept significant tradeoffs. In some cases, these tradeoffs may be acceptable, in other cases maybe not. Furthermore, and importantly, society has invested trillions in infrastructure that efficiently and cost effectively moves liquid hydrocarbons all over the world. It seems reasonable to suggest that when possible we should seek to take advantage and not obsolete infrastructure unnecessarily. There are currently over a billion vehicles in the transportation sector that use liquid hydrocarbons as the source of energy to provide motive power. Thus, any production of sustainable liquid hydrocarbons (bio-based or synthetic) if compatible with the infrastructure, can smoothly transition by continuously increasing the blend ratio and seamlessly phasing out petroleumbased liquid fuels and phasing in sustainable fuels produced in the new carbon asset-based economy. Then the fueling infrastructure can continue to co-evolve with new vehicle infrastructure that does not fully electrify.

\section{Could we lock away a lot of carbon in carbon fibers and carbon composites?}

Human-made material stocks accumulating in buildings, vehicles, roads, and machinery play a crucial role, albeit less appreciated than energy, in shaping the use of resources..$^{13}$ Along with water, especially for agriculture, steel, aluminum, and concrete materials flow through society in many GT/yr scales. It is intriguing to think about displacing some or much of these materials with new materials made from carbon, such as carbon composites and carbon ceramics. The benefits of carbon fiber and its composites include excellent strength to weight ratio, compared to steel or aluminum. In fact, carbon composites could provide lightweight strong alternatives to both steel and aluminum in vehicles. In transportation, lightweight also translates to energy efficiency and further enables electrification. Additionally, carbon composites work well with other materials (fiber, plastics, metals, wood, and concrete). Imagine if we could economically produce carbon fiber from $\mathrm{CO}_{2}$ mined from the excess in the atmosphere. ${ }^{14}$ That would be an innovation challenge with multiple benefits. 


\section{Opportunities and challenges}

Large-scale industrial conversion of solar energy that transforms $\mathrm{CO}_{2}$ and water into infrastructure compatible hydrocarbon fuels and carbon-based materials is an attractive option to facilitate a smooth and continuous transition, affecting the existing vehicle fleet and co-evolving with the future fleet. However, such an option while certainly possible, still has significant resource, economic, and technical challenges before becoming practical, especially if it is going to achieve scale and be sustainable.

A general examination identifies a number of challenges, such as achieving high solar energy-to-fuel system-level efficiency, low material intensity in solar collection, high material accessibility, and good material durability; limited and no additional arable land use (no competition with food); and low water consumption. Opportunities to meet each of these challenges are already encouraging.

Using the sunlight to re-energize $\mathrm{CO}_{2}$ both directly and in hybrids (with biomass or fossil feedstock's) can produce net lower and ultimately net neutral carbon-based fuels with most of the carbon in the initial feedstock making it into the fuel product. This measure of how much carbon makes it into the product refers to the atom efficiency (the ratio of carbon atoms in the product to the carbon atoms in the feedstock) a desirable goal is to achieve near unit atom efficiency, and thus little to no unintended by product $\mathrm{CO}_{2}$. Such innovations could unite solar energy interests with both fossil fuel and advanced biofuel interests, and could preserve an option for a low (and ultimately negative)-net-carbon future and a smooth transition that maximizes the use of installed infrastructure and potentially any new investments in natural gas. Otherwise, long-lived investments in natural gas today could lead to lock-in unless there is a smooth transition to biogas and/ or synthetic methane.

These opportunities offer significant promise for a platform of technologies that store sunlight and sequester carbon above ground as an energy-dense fuel with affordable economics, closing the carboncycle, and scalable to global demand. Extending these fuelbased technologies to carbon-based materials provides an avenue to lock away carbon above ground while creating tangible societal value. Intriguing opportunities, as mentioned above, include carbon fiber, carbon composites, carbon electrodes, and carbon ceramics.

Despite challenges, there are promising advances already happening and opportunities to leverage developments in related industry segments. By working across stovepipes, we could stimulate sustainable economic growth, create many high-quality jobs, and produce viable and scalable solar alternatives to crude oil as the feedstock and carbon-based material alternatives to $\mathrm{CO}_{2}$ intensive steel and concrete.

\section{Acknowledgments}

I wish to acknowledge many useful conversations related to this opinion piece with Professors Gary Dirks, Elisa Graffy, Klaus Lackner, and Bruce Rittmann; and Drs James E Miller and Ivan Ermanoski.

\section{Conflicts of interest}

I have no financial conflict of interest.

\section{References}

1. The Paris Climate Agreement is to achieve the goal of keeping the rise in global average temperatures below 2 degrees Celsius $\left({ }^{\circ} \mathrm{C}\right)$, relative to preindustrial levels. By deriving a correlation between temperature and $\mathrm{CO}_{2}$ concentration in the atmosphere, suggests that we must then keep the $\mathrm{CO}_{2}$ concentration below $450 \mathrm{ppmv}$. Alternatively, noting that the temperature rise is approximately linear in total $\mathrm{CO}_{2}$ emissions since pre-industrial provides a budget of less than $\sim 3000 \mathrm{GT}$. We have already emitted 2200 GT against that total budget.

2. Energy and Climate Change. International Energy Agency. France: IEA Publication; 2015. pp. 200.

3. Lackner KS, Brennan S, Matter JM, et al. The urgency of the development of $\mathrm{CO}_{2}$ capture from ambient air. Proc Natl Acad Sci USA. 2012;109(33):13156-13162.

4. Direct Air Capture of $\mathrm{CO}_{2}$ with Chemicals. A Technology Assessment for the APS Panel on Public Affairs. 2011.

5. Stop emitting $\mathrm{CO}_{2}$ or geoengineering could be our only hope. The Royal Society, UK; 2009.

6. http://carbon.xprize.org/

7. Steinfeld A, Stechel EB. Does the $\$ 20$ Million Carbon XPRIZE Exclude Solar Technologies? Energy Technology. 2017;5:773-774.

8. Dukes JS. Burning Buried Sunshine: Human Consumption of Ancient Solar Energy. Climate Change. 2003;61(1-2):31-44.

9. Boyd E, Boykoff M, Newell P. The 'New' Carbon Economy: What's New? Antipode. 2011;43(3):601-611.

10. K Anderson, G Peters. The trouble with negative emissions. Science. 2016;354(6309):182-183.

11. KS Lackner. The promise of negative emissions Science. Science. 2016;354(6313):714.

12. Stechel EB, Miller JE. Re-energizing $\mathrm{CO}_{2}$ to fuels with the sun: Issues of efficiency, scale, and economics. Journal of CO2 Utilization. 2013;1:2836 .

13. Fridolin K, Dominik W, Christian L, et al. Global socioeconomic material stocks rise 23 -fold over the 20th century and require half of annual resource use. PNAS. 2017;114(8):1880-1885.

14. Mike O. Researcher Demonstrates How to Suck Carbon from the Air, Make Stuff from It. USA: MIT Technology Review; 2015. 